\title{
CLASIFICACIÓN NOMINAL, CONCORDANCIA Y PRONOMBRES
}

\author{
José Ma GARCíA-Miguel \\ (Universidade de Vigo)
}

\begin{abstract}
This work revises some properties of gender and noun classes, within the broader phenomena of noun classification. The main points of the paper are: 1) the distinction of grammatical noun classification from lexical classification of referents; 2) the rypology of noun classification (classifiers, noun classes and gender) in reiation to a continuum of grammaticalization; 3 ) the revision of the possibilitics of agreement as the main formal outcome of gender and noun classes; and 4) the discourse propertics of gender agreement, which leads us to conclude that some gender-like properties of pronouns differ from gender agreement: some pronoun systems are sensitive only to referent properties, while gender systems always refer to grammatical properties of nouns.
\end{abstract}

\section{La clasificación nominal}

Una de las funciones básicas del lenguaje es la de la organizar la experiencia y esa organización se basa en la clasificación o categorización de entidacies y situaciones por medio de los signos de la lengua. Cuáles son los principios generales que utilizan las Ienguas o los seres humanos para realizar esa calegorización es un problema central de las ciencias cognitivas que nos concierne aqui sólo marginalmente. La categorización o clasificación de las entidades del mundo real o imaginado se realiza en las lenguas primariamente mediante los nombres o expresiones nominales. Entre las categorías resultantes, existen relaciones de inclusión que han sido tratadas en semántica lingüística como rclaciones de hiponimia / hipernonimia y que desde los trabajos de Rosch (1978) y otros permiten distinguir entre categorías de nivel básico y categorías superordinadas y subordinadas. Por ejemplo, perro es una categoría de nivel básico 
que tiene a caniche como categoría subordinada y a mamífero y animal como categorías superordinadas. En los niveles más altos de la jerarquía de clasificación existen unos pocos archilexemas muy generales ('persona', 'cosa', 'animal', 'planta', 'idea',...) que en conjunto comprenden casi todo los que categorizamos por medio de los nombres.

Pues bien, al lado de la clasificación que realizan los nombres tenemos la clasificación nominal, es decir, la clasificación de los nombres mismos, como entidades lingüísticas. De los ejemplos citados, los nombres del español caniche, perro, mamifero y animal se clasifican en la lengua española a los efectos de su funcionamiento interno como masculinos, mientras que persona, cosa, planta e idea se clasifican como temeninos. Esta clasificación de nombres se corresponde en parte con categorías de los niveles más altos de la jerarquía de clasificación realizada por nombres (personas o animales de sexo masculino vs. personas o animales de sexo femenino)

Lo importante es que con la clasificación nominal no se clasifican objetos o referentes sino que se clasifican nombres. Se trata de una clasificación de segundo grado o metalinguística' ('clases gramaticales'), que puede estar motivada por la clasificación primaria que realizan los nombres en la lengua objeto ("clases naturales'). Por otro lado, es una clasificación que se manifiesta explícitamente en las formas de palabras relacionadas sintácticamente con esos nombres (perro pequeño, animal pequeño vs. cosa pequeña, persona pequeña) diferenciándose así de cualquicr otro tipo de clasificación de nombres que no tenga manifestación Cormal explícita (por ejemplo, entre nombres abstractos o concretos, comunes y propios, continuos y discontinuos, etc.)

Entenderemos pues por clasificación nominal todo sistema que permita la distribución de los nombres en clases gracias a la existencia de unidades o procesos morfológicos que marquen explícitamente propiedades inherentes del nombre, con tal que la expresión de clase pueda situarse fuera del nombre y que la clasificación, al menos parcialmente, tenga un fundamento semántico. El término clasificación nominal es, por tanto, un término genérico que comprende lo que en los trabajos de linguística aparece, dependiendo sobre todo de las lenguas analizadas, bajo los términos de género, clases nominales y morfemas clasificadores. Veamos algún cjemplo de estos tres tipos de clasificación nominal

En latín existen tres géneros (masculino, femenino y neutro), que se manifiestan en la variación que experimentan en su forma los adjetivos, dependiendo de con qué nombre estén relacionados

\footnotetext{
'El carácter metalinguístico del género es señalado también por Arias Barredo (1990: 115 y 117$) \mathrm{c}$ indirectamente (puesto que lo aplica a todas las categorías morfológicas) por López García (1989: 149)
} 
(1) Latín
a. Bonus filius
«Hijo bueno»
b. Bona filia
«Hija buena»
c. Romanum templum
«Templo romano»

En kirundi, lo mismo que en las otras lenguas bantúes, hay un sistema de clases nominalcs, que se manifiesta mediante prefijos de clase en los nombres y en las palabras que se relacionan sintácticamente con ellos. Hay 17 clases en kirundi, que incluyen la distinción de número singular / plural. Los morfemas que expresan la clase de un nombre aparecen en negrita en los ejemplos y en la glosa un número romano indica de qué clase se trata

(2) Kirundi (Mcl'c\&uk y Bakiza 1987: 286-7)
a. $u-m u-n t u$
$m u-b i$
- I - hombre
I - malo
«Un mal hombre»
b. a-ma-shure
$m a-b i$
-VI-escuela VI-mala
«Malas escuelas»
c. $u-r u-y a b u$
$r u-b i$
-XI-gato XI-malo
«Un gatazo malo»

En tai, como en muchas otras lenguas del sur y este de Asia, un clasificador es una palabra similar a un nombre (que muchas veces puede llegar a usarse como núcieo de la frase nominal) pospuesta obligatoriamente a los numerales que acompañan a los nombres contables y antepuesta opcionalmente a los demostrativos y adjetivos. Hundius \& Kölver (1983) cuentan un total de 64 clasificadores junto con la posibilidad que tienen muchos nombres de repetirse como clasifïcadores de sí mismos. (khan es clasificador de «objetos largos y manipulables», khon de «personas», lûuk de «frutas y objetos tridimensionales / redondos»)

(3) Tai (Hundius \& Kölver 1983:167)
a. rom saăm khan
paraguas tres $\mathrm{CL}$
b. khaw mii lâuk saăm
él liene niño tres $\mathrm{Cl}$
c. khaw sýy mánaaw saăm lâuk
él compró limón tres CL «Él compró tres limones»

\section{Gramaticalización y clasificación nominal}

Uno de los conceptos que mejor puede explicar las diferencias observadas entre las lécnicas de clasificación nominal es el de gramaticalización, que nos permite 
ordenar los tres tipos de técnicas mencionadas en los tres cjemplos anteriores en una gradación de gramaticalización que va desde los clasificadores (como los del ai) hasta cl género (como en Latín) pasando por las clases nominales (como en kirundi)

De los criterios generales que permiten caracterizar el continuum de gramaticalización he recogido en la tabla siguiente algunos de los más destacados, basándome sobre lodo en Bybee (1993), aunque también en aportaciones de Lchmann (1982), Givón (1984), Seiler (1986), Hopper / Traugott (1993), entre otros. La lista, que no es exhaustiva ni está completamente sistematizada, recoge los aspectos más externos de la gramaticalización.

I. Algunas manifestaciones del continuum de gramaticalización

LEXXICO

formas libres y raíces > clíticos (auxiliares, partículas) > alijos, modilïcación interna

posición libre $>$ relativamente fijada

clases abicrtas $>$ clases cerradas grandes

relativamente aro $>$ muy frecuente

semánticamente rico > más generalizado
$>$ estrictamente fijada

$>$ clases cerradas pequeñas

$>$ obligatorio

$>$ reducido o vacío (inmolivado)
GRAMATICAL

De acuerdo con los factores indicados, el género es una técnica altamente gramaticalizada de clasificación nominal, mientras que los clasificadores (como los del tai) son una técnica semigramaticalizada. Las clases nominales (como las del kirundi) ocupan una posición intermedia, aunque más bien próxima al género.

Esa ordenación en un continuum de gramaticalización ya había sido explicitada previamente por quienes han trabajado en este campo (fundamentalmente por Dixon 1986 y por Lehmann 1982)2. El cuadro II, basado en Lchmann (1982: 248), con pequeñas correcciones y añadidos por mi parte, muestra ese continuum de gramaticalización que va desde los clasificadores (que no establecen en principio relaciones de concordancia y forman una clase semiabierta) hasta la existencia de clases formales de nombres que no determinan relaciones de concordancia y generalmente apenas sin motivación semántica. Los fenómenos que nos interesan en este trabajo son los relacionados con la concordancia regida por nombres según su clase o género.

\footnotetext{
2 Como parte del proyecto UNITYP (dirigido por Seiler y en el que también trabajó Lehmann), Sciler (1986) silúa las técnicas de clasificación en la dimensión de aprelensión, entendida como el proceso de captación y representación linguística de los objetos, y correlaciona el grado de gramaticalización con el grado de indicatividad de la técnica correspondiente, es decir, con el proceso que capta los objetos señatándolos décicamente e individualizándolos. Más adelante aludiré a la relación entre clasificación nominal y ancítora
} 
II. Técnicas de clasificación nominal y gramaticalización

\begin{tabular}{|c|c|c|c|}
\hline $\begin{array}{l}\text { Proceso } \\
\text { gencral }\end{array}$ & $\begin{array}{l}\text { clasificación } \\
\text { sintáctica }\end{array}$ & concordancia & $\begin{array}{l}\text { clasificación } \\
\text { morfológica }\end{array}$ \\
\hline $\begin{array}{l}\text { proceso } \\
\text { especílico }\end{array}$ & $\begin{array}{c}\text { clasificador } \\
\text { (determinante / } \\
\text { posesivo / numeral) }\end{array}$ & clasenominal & $\begin{array}{l}\text { clase flexiva } \\
\text { (declinación) }\end{array}$ \\
\hline $\begin{array}{l}\text { clemento } \\
\text { clasificatorio }\end{array}$ & $\begin{array}{l}\text { Clítico } \leftrightarrow \\
\text { aglutinado }\end{array}$ & aglutinado $\longleftarrow$ afijo & $\begin{array}{l}\text { unidad sub- } \\
\text { morfémica }\end{array}$ \\
\hline $\begin{array}{l}\text { motivación } \\
\text { semántical }\end{array}$ & \multicolumn{3}{|c|}{ máxima $<\longrightarrow$ mínima } \\
\hline $\begin{array}{l}\text { gramatica- } \\
\text { lización }\end{array}$ & mínima $<$ & & $\rightarrow$ máxima \\
\hline
\end{tabular}

Lo indicado hasta ahora sobre técnicas de clasificación y elementos clasificatorios es coherente además con una correlación fuerte (pero no estricta) entre las técnicas de clasificación y la tipología clásica de lenguas de base morfológica (cf., por ejemplo, Dixon 1986: 109). En lenguas aislantes (como el tai, chino, victnamita) encontramos un amplio conjunto de clasificadores y difícilmente podríamos encontrar en esas lenguas técnicas que presupongan la utilización de palabras morfológicamente complejas. En las lenguas aglutinantes (como las bantúes) encontramos las llamadas 'clases nominales'; pero en bastantes lenguas aglutinantes (como las fino-ugrias, las altaicas, el quechua o el vasco) no hay ningún sistema gramaticalizado o semi-gramaticalizado de clasificación nominal ${ }^{3}$. Finalmentc, to que suele entenderse por género es típico de lenguas fusionales como las indocuropeas.

Una de las diferencias más llamativas entre los diferentes sistemas de clasificación nominal está en el número de clases. Que el número total de clases sea mayor o menor depende primariamente del grado de gramaticalización alcanzado por el sistema de clasificación, con una variación que va desde un mínimo de dos clases (como en muchos sistemas de género) a varias decenas o algún centenar de clasilicadores en las lenguas con esta técnica lingüística ${ }^{4}$. Como ya he indicado cntre 'género' y 'clases nominales' hay menos diferencias esenciales que diferencias de tradición lingüística. No obstante, los sistemas que suelen recibir el nombre de 'género' varían entre 2 y 4 ó 5 términos en oposición; los que suelen recibir el nombre de 'clases nominales' varían entre 2 , con tal que no opongan

\footnotetext{
${ }^{3}$ Renault (1987) obtiene de la confrontación del finés y el turco con el latín y el ruso la conclusión de que es imposible una lengua aglutinante que posea la categoría género. Sin embargo, él habla de las lenguas bantúes como si no fueran lenguas aglutinantes.

'En los recuentos de clasilicadores que incluyen 'nombres de medida' se llega a varios centenares. Véanse diferentes perspectivas sobre los clasificadores en Allan (1977), Craig (1992) y Croft (1994)
} 
'masculino' y 'femenino', y un par de decenas (en Fula, una lengua nigercongoleña hablada en Nigeria, se reconocen 20 clases $)^{5}$.

Las diferencias en el grado de gramaticalización del género frente a los clasificadores se manifiesta también en la indeterminación en el tamaño de los sistemas de clasificadores nominales: a diferencia de lo que ocurre en los sistemas de género o clases nominales (bastante estables), el número de clasificadores utilizados en una lengua está sometido a gran variación diacrónica, diastrática y diafásica. Los registros más formales suelen incluir un conjunto más amplio de clasilicadores, mientras que el habla informal, y en mayor medida el habla infantil, utilizá un conjunto más reducido de clasificadores más generales ${ }^{6}$

Como vemos, las lenguas pueden diferir bastante en las lécnicas de clasificación nominal utilizadas y en el repertorio correspondiente; pero, además, ni siquiera el concepto mismo de clasificación nominal es un universal lingüístico. Ya he mencionado la existencia de lenguas sin ningún tipo de clasificación nominal: húngaro, finés, vasco, georgiano, quechua, lenguas túrcicas ${ }^{7}$. Por supuesto, todas estas lenguas pueden recoger en el léxico nominal oposiciones que en otras lenguas se corrclacionan con diferencias de género ${ }^{8}$. La oposición léxica 'hombre' / 'mujer' es probablemente un universal lingüístico (Croft 1994: 162), pero no lo es, ni mucho menos, la oposición de género masculino / femenino.

\section{Género, clases nominales y concordancia}

De los tres fenómenos que se comprenden bajo el concepto de clasificación nominal, lo que define al género y las clases nominales como técnicas

\footnotetext{
${ }^{5}$ Más allí de eso, Dixon (1968: 167-168) cita dos lenguas que muestran concordancia de los modificadores con el nombre y que tienen una de ellas (la lengua austronésica nauruano) 40 clases y la orra (la lengua indopacífica de Nueva Guinea nasiói) por lo menos 115 marcadores de clase distintos. Según Cook \& Criswell (1993), en koreguaje, una lengua tucana hablada en Colombia, existen unos 30 morlemas clasificadores que se sufijan al nombre y, concordando con él a los adjetivos y demostrativos que lo acompañan (por ejemplo joo-wi iha-wi «canoa grande»; sukhi-ñi iha-ñni «átbol grande»). No obstante, debe tenerse en cuenta lo dicho sobre los límites borrosos entre género, clases y clasificadores. "Cf. Erbaugh (1986), sobre el chino y DeLancey (1986) sobre el Tai.

${ }^{7}$ El turco conserva algunos clasificadores numerales (Lewis 1967:80-1): kisi 'persona', bas 'cabeza' (para ganndo y vegetales), el 'mano' (para armas de fuego y cartas), tane 'grano' (para cosas en general). El turco otomano (variante 'clásica' con muchos elementos arabes y persas utilizada en cscritos oliciales hasta la llegada de la república en 1922) contaba con alguno más: aded 'número' (equivalente de tame), kita 'picza' (con libros, documentos, barcos, campos), pare 'pieza' (con artillería, barcos, pucblos)

"Por ejemplo en húngaro (Moreno 1991: 182-3): orvos 'médico' / orvosnö 'médica' lorvosné 'mujer del médico'; farkas 'lobo' / nöstenyfarkas 'loba'; galamb 'paloma'/ himgalamb 'palomo' (nö 'mujer'; nösteny 'hembra', him 'macho'). En turco (Lewis 1967: 25): aygir «caballo» / kisrak «yegua»; kardes «hernano o hermana»/ kizkardes «hermana»
} 
gramaticalizadas de clasificación nominal es la concordancia, entendida como covariación obligatoria en la forma de palabras relacionadas sintácticamente con el nombre.

Los principalcs trabajos sobre el género y sobre clases nominales ${ }^{9}$ toman la concordancia como criterio de identificación para concluir que el género (como el del latín, el español u otras lenguas indoeuropeas) y las clases nominales (como las lenguas bantúes) son esencialmente el mismo fenómeno y que es ése el principal criterio que permite distinguirlos de los clasificadores.

Las clases nominales y el género comparten la propiedad de la concordancia y forman un continuum en el que intervienen como criterios potencialmente diferenciadores: el número de clases, el grado de fusión de los morfemas de clase, el grado de motivación semántica de las clases, la movilidad derivativa de los nombres respecto de las clases, etc... En esta gradación que permite situar en un cxtremo las clases nominales bantúes y en el otro el género indoeuropeo, quedan en medio lenguas estructuralmente similares a las que se les ha atribuido en la tradición bien un sistema de género bien un sistema de clases. Lo de menos son los critcrios especílicos de clasilicación, pero hay quien reserva el término de género para los sistemas que utilizan el sexo como criterio de clasificación. Sintáclicamente, en cambio, no hay diferencias sustanciales entre clases nominales y género. Cuando no establezca oposición entre los dos conceptos utilizaré el término géncro para referirme a ambos.

Dado que lo específico del género es la concordancia, en el caso de que la clasilicación se refleje de alguna manera en la forma del nombre además de en los adyacentes puede y suele haber discordancias entre unas marcas y otras. Esto obliga a distinguir entre género / clase nominal y clases formales (que pueden dar lugar a clases flexivas o declinaciones) en que pueden agruparse los nombres. Por cjemplo, en latín fagus es femenino a pesar de pertenecer a la $2^{a}$ declinación. De la misma manera, en español mano es femenino a pesar de acabar en -o. Fenómenos similares podemos encontrar en otras lenguas, incluidas las que más regularmente marcan la clase gramatical (como las lenguas bantúes).

Ante la posibilidad de discrepancias, debe quedar claro que lo que estamos considerando como el género o clase de un nombre concierne exclusivamente a las posibilidades de concordancia, y no a las marcas morfológicas presentes en el nombre o junto a ćl, por mucho que pueda llegar a existir una correspondencia fuerte entre una y otra.

Habiendo establecido la concordancia como característica de género y clases nominales pasamos a preguntarnos qué es lo que puede concordar en géncro con un nombre y encontraremos que las lenguas varían desde la posibilidad de que

\footnotetext{
${ }^{9}$ Por ejemplo, Hjelmslev 1956:280, Ibrahim 1973:63, Dixon 1968:160, Corbett 1991
} 
concuerde con el nombre un solo tipo de palabra o partícula, como en dyirbal, hasta la posibilidad de que concuerde casi todo lo que hay en la cláusula donde aparece esc nombre. En las lenguas bantúes, como vemos en (4), casi todas las palabras de Ia cláusula pueden llegar a repetir en la concordancia el prefijo de un nombre

(4) Suahili (cf. Ashton 1941: 58 y 336)
a. wa-geni wa-ke wa-wili wa-lifika jana
2-invitados 2-suyos 2-dos 2-llegaron ayer
«Sus dos invitados llegaron ayer»
$\begin{array}{cllll}\text { b. } v i \text {-kombe } & v i \text {-wili na } v i \text {-sahani } & \text { vy-ake } & \text { vi-mevunjika } \\ \text { 8-tazas } & \text { 8-dos y } & \text { 8-platos } & 8 \text {-suyos } & \text { 8-se han roto }\end{array}$
«Dos copas y sus platos se han roto»

Un poco más arriba he aludido a que la concordancia se establece entre elementos relacionados sintácticamente. Esto nos da como posibles elementos concordantes en género los que se deducen del cuadro III, que recoge las principales posiciones estructurales en las que encontramos nombres y frases nominales, marcados en el cuadro en negrita con el subíndice $\mathbf{r}$ que corresponde a su clase, y junto a ellos los elementos potencialmente concordantes marcados con el subíndice $\mathbf{d}$ que señalaría morfológicamente la clase $\mathbf{r}$ del nombre o frase nominal regente. Las posibilidades del cuadro se han contrastado con el estudio de Lehmann (1982), del que tomo la distinción entre 'concordancia interna' y 'concordancia externa', y con la enumeración no sistematizada de Corbett (1991: 106-114):

III. Elementos potencialmente concordantes en género ( $\mathrm{r}=$ género regente; d=género regido)

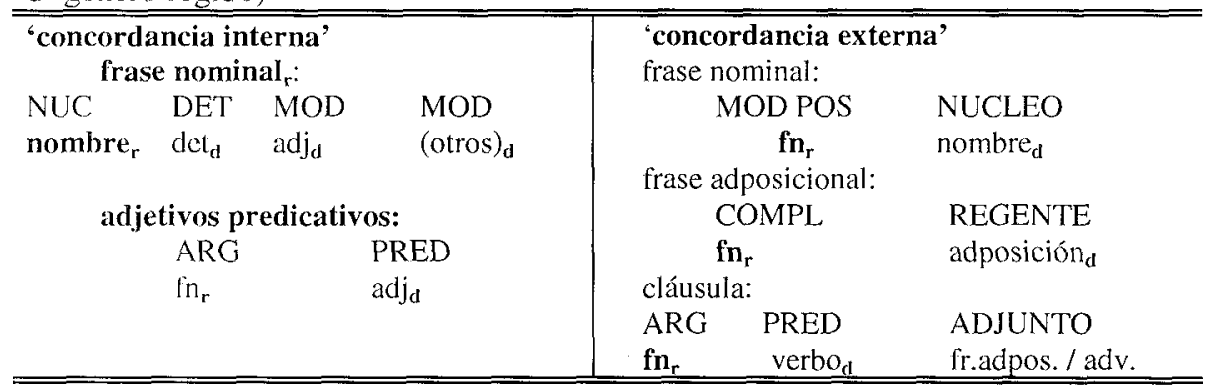

Tenemos en primer lugar la 'concordancia interna', es decir, la concordancia con elementos que forman parte de la frase nominal que rige la concordancia (la frase de la que cs núcleo el nombre clasificado, si lo hay). Por ejemplo, en (5) el 
sustantivo augurio / noticia concuerda con el demostrativo ese / esa y el adjetivo calificativo mal/ malce, que forman parte de la misma frase nominal.

\section{(5) Español}

a. Ese mal augurio no parece fundado

b. Esa mala noticia no parece fundada

\section{Pueden manifestar concordancia interna:}

a) Los determinantes: artículo, demostrativos como el Dyirbal en (6), posesivos personales, numerales como el suahili en (4), e indefinidos

(6) Dyirbal (Dixon 1982: 161)

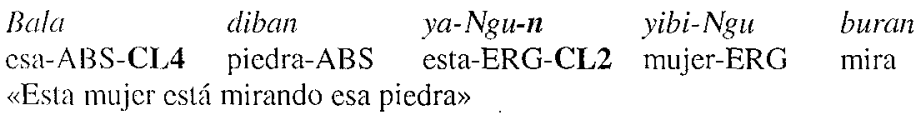

b) Los adjetivos calificativos que funcionan como modificadores en la frase nominal (adjetivos atributivos). Sirva de ejemplo el del español de (5), que muestra concordancia con demostrativo y adjetivo calificativo. A veces, la concordancia de determinantes y adjetivos modificadores puede ser variable. En alcmán, cl género lo manifiesta primariamente el artículo definido y sólo si falta éste lo manifiesta cl adjetivo calificativo.

\section{(7) Alemán}

a. der große Erfolg (masc.) / die große Stadt (fem.) / das große Fenster (neut.)

b. ein großer Erfolg (masc.) / eine große Stadt (fem.) /ein großes Fenster (neut.) el-un gran éxito la-una gran ciudad la-una ventana grande

c) En tcrcer lugar, pueden concordar en género, aunque más raramente, otros modificadores en la frase nominal, constituidos típicamente por frases adposicionales o frases nominales oblicuas. En algunas lenguas, como la lengua caucásica chamalal, los modificadores posesivos concuerdan en género /clase con el núcleo de la frase. También pueden concordar morfemas asociativos de relación (suahili y lenguas bantúes) y otras frases adposicionales dentro de la FN

(8) Chamalal (Lehmann 1982: 209)
a. hek'wa-sisto wac hombre-de-CLl hermano
«El hermano del hombre»
b. hek'wa-sis-i jac hombre-de-CL2 hermana
«a hermana del hombre»
c. hek'wa-ist-b
c'atv
hombre-de-CL 3 caballo
«El caballo del hombre» 
$\begin{array}{ll}\text { d. hek'wa-s, } \ell-l & \tilde{l} c a \\ \text { hombre-de-CL4 } & \text { queso }\end{array}$

Los adjetivos predicativos se sitúan, por definición, fuera de la frase nominal. Sin cmbargo, en lo que afecta a la concordancia, los adjetivos predicativos suelen comportarse del mismo modo que los adjetivos atributivos, es decir, igual que la concordancia interna, como vemos en español en (5).

Frente a la concordancia interna, tenemos lo que Lehmann llama 'concordancia externa', la concordancia con elementos que no forman parte de la frase nominal, si la hay, que rige la concordancia.

Son manifestaciones de la concordancia externa

a) La relación inversa de la que he ejemplificado con el chamalal: que el núcleo de una frase nominal (objeto poseído) concuerde la frase nominal que la modilica como poseedor. Es como si en (8c) se marcara en la palabra 'caballo' la clase a la que pertenece 'hombre', en vez de indicar junto a 'hombre' la clase a la que pertenece 'caballo'. También pueden concordar en género con una frase nominal las adposiciones que la rigen dentro de las frases adposicionales. En alguna lengua caucásica como el abjaso podemos encontrar muestras de este tipo de concordancia. Sin embargo, tanto cuando una adposición señala características gramaticales de su complemento como cuando lo poseído señala características gramaticales del poseedor, lo habitual es que se trate de índices de número y persona pero más raramente de clase o género .

b) En la cláusula, es frecuente que el predicado (verbo) concuerde con el sujeto y otras funciones centrales, pero también es más raro que lo haga en género. En ruso, cl verbo concuerda en género con el sujeto en pasado como vemos en (9). En suahili, el verbo puede concordar con sujeto y objeto, como vemos cn (10), donde cl verbo contiene morfemas que indican que el sujeto pertenece a la clase I y el objeto a la clase 7

(9)Ruso (Lehmann 1982: 211)

żurnal gorel (masc.) (neut.)

el periódico ardió

(10)Suahili (Ashton 1941: 45)

Hamisi a-me-ki-leta

Hamisi 1-T-7-traer

kniga gorela (fem.) - _.. pis'mo gorelo

el libro ardió

la carta ardió

ch-akula

7-libro «Hamisi ha traído el libro»

c) Muy raro es que un constituyente argumental de una cláusula llegue a concordar con constituyentes distintos del predicado (con los «adjuntos»). No obstantc, Moreno Cabrera recoge de Lüdke el ejemplo de (11), perteneciente a 
un dialecto del italiano, en el que el género del sujeto hace variar la forma de un complemento locativo.

(11)Italiano (Habla de Ripatransone) (Moreno 1991: 183)

$\begin{array}{cllll}\text { a. } l-\boldsymbol{u} & \text { frekki } & \grave{e} \text { it-u } & \text { a } & \text { rom-a } \\ \mathrm{cl} & \text { niño } & \text { ha ido } & \text { a } & \text { Roma } \\ \text { b. l-e frëkine } & \grave{e} \text { it-a } & \text { a } & \text { rom-e } \\ \text { la niña } & \text { ha ido } & \text { a } & \text { Roma }\end{array}$

En csia cnumeración de elementos potencialmente concordantes en género hay dos omisiones que merecen un pequeño comentario. Por un lado, no he considerado la posibilidad de que una frase nominal concuerde en género con otra porque cada frase nominal sólo manifiesta un género determinado por propiedades inherentes de la frase nominal $y$ en caso de que dos frases nominales relacionadas sintácticamente (por cjemplo en relaciones apositivas) coincidan en género se trata de una simple coincidencia y no de concordancia.

La otra omisión, más importante pero no completamente desligada de la anterior, es la de los pronombres, aunque no ignoro la afinidad entre concordancia y anáfora, ni el hecho de que en una relación anafórica el pronombre puede y suele indicar cl género del antecedente. De hecho, Lehmann (1982) incluye los pronombres entre los elementos que manifiestan concordancia externa y Hjelmslev (1938) deline el género y número por contraer rección ('dirección néxica') simuitáneamente homonexual y heteronexual, es decir, por manifestarse tanto en relaciones que existen en el interior de un 'nexo' o cláusula («concordancia») como cn relaciones que se contraen entre unidades de nexos diferentes (como suele ocurrir entre pronombre y antecedente). Hablaré más adelante de la relación entre los pronombres y la clasificación nominal.

Para cerrar este apartado, indicaré es posible formular algo bastante próximo a una 'jerarquía de concordancia' (entendida como facilidad para concordar en géncro / clase con un nombre), que he resumido en el cuadro IV

IV. Tendencias en la concordancia de género

concordancia interna > concordancia externa

concordancia interna:

determinantes $>$ adjetivos atributivos $>$ adjetivos predicativos $>$ otros

modificadores nominales

concordancia cxterna:

verbos $[\rightarrow$ sujeto $>$ objeto $]>$ otros constituyentes 
La mayor facilidad para que la concordancia de género se asocie con el dominio interno es observada por Lehmann (1982: 259) y se corresponde con uno de los universales de Greenberg (1963) relativos a la concordancia de género:

31: Si el verbo concuerda en género con el sujeto o el objeto, el adjetivo también concuerda con el nombre

En cambio, la posición relativa de los elementos que muestran concordancia interna, on particular la posición relativa de determinantes y adjetivos ${ }^{10}$, puede plantear mayores problemas.

\section{Géneros regentes y géneros regidos}

Hemos visto que el género es una propiedad de los nombres que se comprueba, mediante la concordancia, en palabras que no son nombres. El criterio de clasilicación de los nombres serán sus pautas o esquemas de concordancia. Siguiendo en lo lundamental a Corbett (1989 y 1991: cap. 6), vamos a ver algunos problemas relativos al establecimiento del número de géneros tomando la concordancia como criterio definitorio de la categoría. Volviendo al ejemplo (5) del español, el nombre augurio pertenece a una clase gramatical porque se combina con las formas ese, malo y fundado, mientras que el nombre noticia pertenece a otra clase porque se combina con esa, mala y fundada.

\section{(5)Español}

a. Ese mal augurio no parece fundado

b. Esa mala noticia no parece fundada

Pero tenemos que distinguir entre las clases en que agrupamos los nombres de una lengua tomando como criterio la concordancia (la clase a la que perteneco augurio frente a la clase a la que pertenece noticia) y las opciones que manifiesta un término concor-dante dado (por ejemplo, la oposición fundado / fundada). A lo primero lo llama Corbett (1989 y 1991: 151) controller genders l'géneros regentes'], a lo segundo lo llama target genders ['géneros regidos']. Tendremos, pues tantos géneros regentes como clases puedan definirse en el nombre a partir de las pautas de concordancia y tantos géneros regidos en una categoría dada como

\footnotetext{
10) A favor de la ordenación propuesta están lenguas como el dyirbal que manifiestan el género / clase nominal en los dé́cticos pero no en los adjetivos. La preeminencia de los determinantes en esa jerarquía parece estar de acuerdo con el funcionamiento de los clasificadores $y$, por tanto, con una continuidad cntre género y clasificadores. También parece estar de acuerdo con el origen de los marcadores de género en los nombres (Greenberg 1978), mediante la aglutinación de determinantes
} 
formas difcrentes cn covariación con el nombre regente.

V. Géneros regentes y géneros regidos en correspondencia biunívoca

$\begin{array}{cllll}\text { Géneros regentes } & \text { Adj. singular } & \text { Adj. plural } & \text { Dem. sing. } & \text { Dem. PI. } \\ \text { I augurio(s) } & \text { mal, fundado } & \text { malos, fundados } & \text { ese } & \text { esos } \\ \text { Il noticia(s) } & \text { mala, fundada } & \text { malas, fundadas } & \text { esa } & \text { esas }\end{array}$

En principio, esperamos una correspondencia biunívoca entre las clases de nombres (los géneros regentes) y las opciones de género en los términos concordantes (los géneros regidos). Esperamos, por ejemplo, que los nombres de género masculino concuerden con adjetivos y demostrativos en masculino y los nombres de género femenino concuerden con adjetivos y demostrativos en femenino, y csto independientemente del tipo y función del adjetivo o de si el elemento concordante está en singular o en plural.

Sin cmbargo, esá correspondencia biunívoca puede fallar y llevarnos a una situación en la que cl número de géneros nominales ('regentes') sea distinto del de las opciones disponibles en todos o en parte de los elementos concordantes ('géneros regidos'). En mi opinión, existen tres posibilidades básicas, que pueden combinarse de forma compleja":

a) En primer lugar puede ocurrir que el número de distinciones (de géneros regidos) se reduzca cn ciertos contextos. Esto puede ocurrir bien por un sincretismo más o menos ocasional (en español hay muchos adjetivos como azul que no manifiestan oposición de género; en latín las distinciones de género de presentan de distinto modo en los adjetivos de la $3^{\circ}$ declinación que en los de la $1^{a}$ y $2^{a}$ ) o en una neutralización de la oposición de género en contextos gramaticalmente especilicables.

VI. Sincretismo o ncutralización de género en parte de los elementos concordantes (EC)

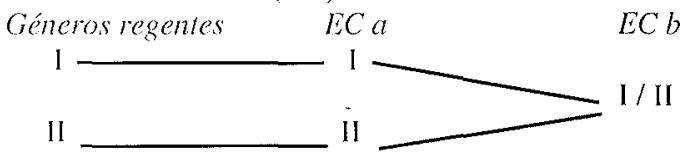

Entre las tendencias generales que acompañan a esta reducción de distinciones de géncro, la más signilícaliva, por mostrar la estrecha relación que existe entre el género y el número es que suele haber menos opciones de género en plural que en singular. Esto responde a otro de los universales de Greenberg (1963)

\footnotetext{
"Vid. el análisis exhaustivo de lo que puede dar de sí la distinción entre 'controller' y 'target' 'genders' en Corbett (1989 y 1991: caps. 6 y 7 )
} 
37: En el singular nunca hay menos distinciones de género que en otros números (pl., du. )

Algunas lenguas que ejemplifican esta reducción de géneros en el plural con respecto al singular son (Corbett 1991: $\$ 7.1)$ : hausa $(2 \rightarrow 1)$, alemán $(3 \rightarrow 1)$, seneca $(3 \rightarrow 2)$, tamil $(3 \rightarrow 2)$, chamalal $(5 \rightarrow 2)$, fula $(20 \rightarrow 5)$, etc.

b) La segunda posibilidad que vamos a ver es que exista una correspondencia no univoca entre los opciones que encontramos en diferentes condiciones gramaticales, to que puede llevarnos a que exista mayor número de géneros nominales [géneros regentes] que de opciones disponibles como géneros regidos. Lo veremos con un ejemplo: En rumano hay tres clases de nombres (tres "controller gender') a pesar de que el número máximo de opciones ('target gender') en cl adjctivo es dos, porque las opciones en el singular no se corresponden unívocamente con las opciones en el plural y obtenemos tres pares singular / plural

(12) Rumano (Corbett 1991: 150-1)

a. Barbatul e bun - barbatii sint buni ['masc.']

«El hombre es bueno / los hombres...»

b. Scaunul e bun - scaunele sint bune ['neutr']

«a silla cs bucna / las sillas son buenas»

c. Fata bună $\quad$ - fetele sint bune ['fem.]

«a chica es buena / las chicas...»

VII. Géneros en rumano (según Corbett 1991: 152)

Adjetivo singular

Géneros Nominales

Adjetivo plural

('regentes')

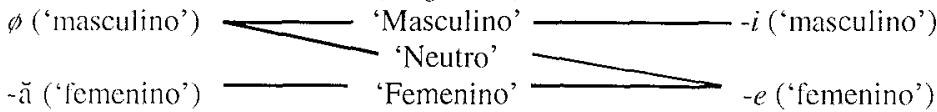

Lo que nos muestra el sistema rumano es que tendremos un género nominal por cada pauta de concordancia, es decir por cada combinación de opciones en los clementos concordantes que se asocie con una clase de nombres (en el ejemplo, hay de cada vez dos opciones, pero en total tenemos tres combinaciones singular / plural y por tanto tres géneros nominales) ${ }^{12}$. Eso no quiere decir que quepa cualquier combinación entre las opciones que manifiesta diferentes elementos concordantes. Carstairs-McCarthy (1994) ha formulado una restricción sobre la

\footnotetext{
12 La altcrnativa a esta concepción del género desarrollada por Corbelt es renunciar a establecer un sistema único de clases nominales y recurrir a un polisistematismo que permite clases diferentes a partir de condiciones o criterios gramaticales diferentes.
} 
morlología del género ${ }^{13}$ que afecta al número máximo de 'géneros regentes' que caben en un sistema con sincretismos de este tipo:

'No blur principle for gender': «Within any set of inflectional affixes which realize the same paradigmatic cell (apart from difference in gender) on a gender target, no more than one can fail to identify the controller gender of the controller unambigously» (Carstairs-McCarthy 1994: 770)

El principio -que, según Carstairs-McCarthy, tiene algunas excepciones sólo en algunas lenguas con género explícito ('overt gender') en el nombre- impide que, por cjemplo, exista un cuarto género en rumano con nombres femeninos en singular y masculinos en plural; pues entonces a la ambigüedad del masculino cn singular se añadiría la del femenino y a la ambigüedad del femenino en plural se añadiría la del masculino.

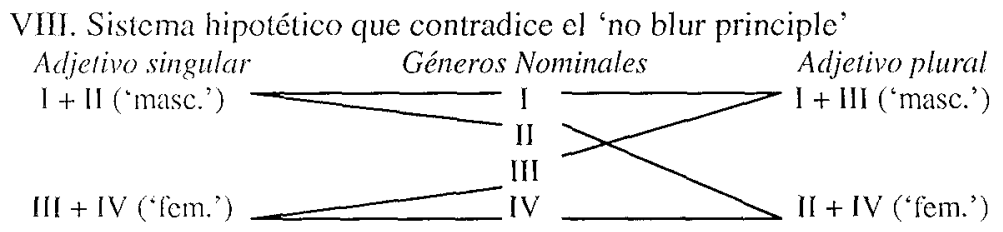

c) Si, como vemos, cabe la posibilidad de que existan más géneros nominales controladores que opciones disponibles en los elementos concordantes, también cabe la posibilidad contraria, que existan mayor número de opciones en algún término concordante que géneros de nombres. Esto es posible si existen lo que Ilama Corbett 'géncros no léxicos', es decir, opciones en un término concordante que no admiten combinarse con ningún nombre. Sin ir más lejos, es lo que ocurre con el artículo neutro en español

\footnotetext{
(13) Español

el mal augurio - la mala noticia - lo malo

IX. El neutro del artículo español como género 'no léxico'

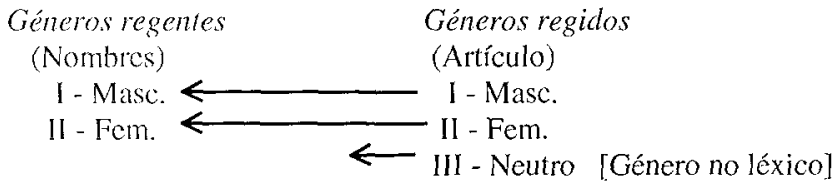

\footnotetext{
${ }^{13}$ La restricción está basada en el 'principio de contraste' y es de naturaleza similar al 'Paradigm Economy Principle' claborado también por Carstairs (1983)
} 
Para terminar este punto, debemos distinguir las posibilidades indicadas, que conciemen a las correspondencias entre géneros de nombres y opciones en términos concordantes, de la existencia de lexemas nominales con más de un esquema de concordancia. Tenemos lo que se han llamado nombres de 'género' comín (el/la artista), que se combinan con más de un género en cualquiera de los términos concordantes, además del cambio de género acompañado de variación morlológica (niño / niña $a^{14}$ ) o de variación puramente léxica (hombre /mujer). En cualquier caso, cl género común no es un género más (una clase más o una opción más) en la lengua, afecta sólo a cómo se distribuyen los nombres en relación con las pautas de concordancia (géneros) y, en su caso, a los procedimientos morfológicos más o menos productivos que permiten a un nombre cambiar de género.

\section{Los pronombres y las distinciones de género}

El último punto que vamos a tocar en este trabajo está relacionado con las funciones discursivas de los sistemas de clasificación nominal. La función básica de los clasilicadores en las lenguas que los poseen no es simplemente agrupar los nombres o sus referentes en clases sino individualizarlos a efectos de enumeración o de identificación en el discurso. Los clasificadores pueden servir para introducir referentes específicos y también para recuperar en las relaciones anafóricas refcrentes ya introducidos en el discurso. Eso no quiere decir que todo recurso anafórico requiera un sistema de clasificación ni siquiera que los clasificadores puedan utilizarse en todas las lenguas que los tienen como recurso anafórico ${ }^{15}$. Pero la clasilicación nominal nos proporciona un sistema de categorización adicional a la categorización realizada por los nombres, pero necesariamente mucho más reducido. Esto pone a disposición de los hablantes un conjunto reducido de opciones que puede ser suficiente para señalar referentes ya activados en la memoria de trabajo y que a la vez puede discriminar entre referentes potenciales.

El género, al ser obligatorio, no puede utilizarse como expresión de especificidad / individualización, aunque sí puede utilizarse una oposición de género para expresar por ejemplo una oposición entre contable y no contable ${ }^{16}$. La

\footnotetext{
${ }^{1-}$ Las oposiciones de este tipo en el nombre tienden a ser vistas como derivativas (lexemas diferentes) y no como llexivas (cf. Aronolf 1994: 182)

15 Denny (1976:130) recoge algunos datos sobre el uso variable de los clasificadores como recurso analórico. Vid. también Downing (1986) y Hopper (1986). Dixon (1986: 109) correlaciona el número de clases y la organización del discurso, a partir del hecho de que el dyirbal, con cuatro clases, recurre a la elipsis; el yidiñ, con una veintena de clasificadores, recurre a los clasificadores en la cohesión referencial.

${ }^{16}$ El danćs, según Johnsen (1992), y el sueco utilizan a veces la oposición común / neutro como contable / no contable. Lo mismo pasa con el neutro de algunos dialectos del asturiano.
} 
principal función de las distinciones de género es la de toda concordancia, establecer la cohesión sintáctica entre las palabras de una secuencia, lo cual permite secundariamente establecer a qué referente es aplicable cada término concordante ${ }^{17}$. En un interesante trabajo, Zubin y Köpcke (1986) proponen una justificación para el numeroso grupo de palabras referidas a objetos que en alemán tienen un género distinto del neutro. Obscrvan una fuerte correlación entre la utilización del masculino o el femenino y lo que Rosch (1978) llama categorías de nivel básico, mientras que los nombres superordinados (los archilexemas correspondientes) son neutros:

(14) Alemán (Zubin y Köpcke 1986: 147 y 166)

\begin{tabular}{|c|c|}
\hline a. das Instrument $(\mathrm{N})$ & : die Guitarre $(\mathrm{F})$ \\
\hline 'instrumento' & : 'guitarra' \\
\hline b. das Werkzeug $(\mathrm{N})$ & : der Hammer $(\mathrm{M})$, die Säge $(\mathrm{F})$ \\
\hline 'herramienta' & 'martillo', 'sierra' \\
\hline c. das Mobelstiick (N) & der Tisch $(\mathrm{M})$, die Lampe $(\mathrm{F})$ \\
\hline 'mucble' & : 'mesa', 'lámpara' \\
\hline d. das Wissen $(\mathrm{N})$ & : die Kunst $(\mathrm{F})$, die Linguistik $(\mathrm{F})$ \\
\hline $\begin{array}{l}\text { 'conocimiento' } \\
\text { c. das Geld }(\mathrm{N})\end{array}$ & $\begin{array}{l}\text { 'arte', 'lingüística' } \\
\text { der Frank }(\mathrm{M}) \text {, die Lira }(\mathrm{F})\end{array}$ \\
\hline 'dincro' & : 'franco', 'lira' \\
\hline
\end{tabular}

La razón de este comportamiento (Ibid.: 173-4) está en la resolución del conflicto cntre la motivación máxima del sistema de género y la utilización máxima del sistema con carácter diferencial (al servicio de la función comunicativa incrementando el carácter distintivo de las referencias anafóricas). En otras palabras, de lo que se trata es de asignar géneros variados a las palabras pertenecientes al mismo dominio para facilitar así su recuperación anafórica.

La contribución de la concordancia de género a la identificación de referentes incluso permitc en algunas lenguas la ausencia de nombre en el núcleo de la frase nominal, como podemos comprobar tanto en (15) como en (16), frente a la necesaria utilización de la proforma one en inglés, una lengua sin distinciones de género en la frase nominal

(15) Suahili (Ashton 1941: 52)

a. Lete visu vikubua

«Trae (los) cuchillos grandes»

b. Lete vikubuat

(16) Español «Trae (los) grandes»

\footnotetext{
${ }^{17} \mathrm{Cl}$. Arias Barredo (1990: 119): «La concordancia no es sólo un fenómeno de forma expresiva, sino que implicar el manejo de la referencia interna o intratextual, que, de alguna manera, compromete la referencia linguística (...) La determinación sintagmática del género no es otra cosa que una exigencia de la función fórica: (...) el sustantivo proyecta su género en todo el ámbito de la propia referencias
} 


\section{a. Trae los cuchillos grandes \\ b. Trae los grandes}

\section{(17) Inglés}

\section{a. Bring some big knives \\ b. Bring the big ones}

Esta relación entre las distinciones de género y la recuperación de referentes nos lleva a las complejas relaciones entre los pronombres y el género. Martinet (1956: 326), por ejemplo, considera que «es en los pronombres donde la distinción entre los géneros rinde verdaderos servicios» y el mismo año Hjelmslev (1956: 306-7) obscrva que muchas lenguas que han perdido el género en el nombre «lo conservan, sin embargo, en esa posición clave que es el pronombre y, sobre todo, el pronombre anafórico» ${ }^{18}$. Está, además, el problema de decidir hasta qué punto los pronombres representan un caso particular de concordancia $y$, por tanto, hasta qué punio podemos decir que una lengua como el inglés tiene categoría de género, que es lo que defiende Corbett (1991: 12 y 112) al considerar la variación de género en los pronombres como un caso particular de concordancia.

Para aproximarnos a la resolución de este problema vamos a echarle un vistazo a la jerarquía de concordancia propuesta por Corbett en la cual, según avanzamos hacia la derecha más probable es que la llamada 'concordancia semántica', es decir, que la clección de clase se base en características del referente y no en el género del antecedente o del nombre regente ${ }^{19}$.

\section{La jerarquía de concordancia (Corbett 1991: 226)}

modilicadores $<$ predicados $<$ pronombres relativos $<$ pronombres personales

Las principales implicaciones de la jerarquía de concordancia las comprobaremos en el francés y en el español con palabras en las que hay un posible conflicto entre clase gramatical (género) y clase nocional del referente. Como vemos en (18) y (19), el francés utiliza sistemáticamente el femenino para remitir a palabras como Sainteté o Majesté, aunque tengan referente masculino. Sin embargo, en el extremo derecho de la jerarquía, cabe también el pronombre personal masculino, como vemos en (18d)

\footnotetext{
18 Además, «en el pronombre interrogativo una distinción entre el género animado y el género inanimado, o cntre el género personal y el género no-personal, está en extremo difundida entre las lenguas del mundo fincluso en lenguas como el finés, húngaro, chino, tai, georgiano, <turco>, armenio clásico]» (lbid.)

1") "As we move rightwards along the hierarchy, the likelihood of semantic agreenent will increase monotonically (that is, with no intervening decrease)» (Corbett 1991: 226)
} 
(18) Francés (cjemplos citados por Grevisse apud Corbett 1991: 226-7)

a. Sa Sainteté n'est pas si ombrageuse de s'en formaliser

b. Sa Sainteté, avec laquelle je viens de parler...

c. Votre Majesté partira quand elle voudra

d. Sa Majesté fut inquiète, et de nouveau il envoya La Varenne à son ministre

(19) Francés (La Fontaine: Le loup et l'agneau)

...que Votre Majesté

ne se mette pas en colère;

mais plutôt qu'elle considère

que je me vas désaltérant

clans le courant

plus de vingt pas au-dessous d'Elle;

et que par conséquent, en aucune façon,

je en puis troubler sa boisson

El español, por el contrario, suele utilizar en condiciones similares en masculino, como vemos en (20), pero en el extremo izquierdo de la jerarquía, el femenino es obligatorio para los modificadores internos a la frase nominal

(20) Español (Corbett 1991: 230)

A Su Majestad suprena, el cual está muy contento aquí en Valencia, $\underline{\text { e }}$ recibieron con muchos aplausos. Él se mostró muy emocionado

Además, el género de los pronombres personales puede variar dependiendo de la distancia con respecto al antecedente

(21) Español (Ortega, citado por Wonder 1978: 19)

La 'hombria' estaba, no en sus personas, sino en torno a ellas: era una mística aureola, un nimbo patético que los circundaba.. Las masas habian creído en ellos

La 'jerarquía de concordancia' de Corbett, que muestra cómo se manifiesta en las Ienguas la dicotomía entre concordancia formal (o concordancia 'gramatical') y concordancia scmántica (o concordancia 'natural' o concordancia 'ad sensum') recoge fundamentalmente los dos hechos siguientes (cf. Lehmann 1982: 251):

a) En cl interior de la frase nominal (en la 'concordancia interna') no pueden existir contradicciones en las marcas de género: a cada frase nominal sólo puede corresponder un género gramatical

b) A mayor distancia sintáctica mayor es la posibilidad de concordancia scmántica. En la medida en que se pierde la conexión sintagmática directa, se pierde también la covariación formal que la manifiesta. 
En mi opinión, lo que ocurre en los casos de ausencia de concordancia formal es que la selección de género se establece de manera independiente, es decir que en lo que estamos llamando concordancia "natural' o 'nocional' no hay concordancia sino que se selecciona el género con criterios que conciernen exclusivamente al pronombre (o al adjetivo en su caso) y a su relación con la realidad y no con nombres mencionados previamente. Esto es posible en la medida en que el antecedente y el elemento potencialmente concordante estén también sintáctica y textualmentc distanciados como expresiones (potencialmente) referenciales independientes. En suma, la independencia que pueden llegar a mostrar los pronombres en la selección de género es análoga a la que muestran las cadenas referenciales establecidas mediante cohesión léxica, como la que forman en (22) el femenino personas y el masculino converso:

(22) Los que han guardado intimidad con personas que han sufrido eso que se llama conversiones, han podido observar que el converso no ha cambiado de conducta.

A lo anterior se puede añadir una faceta más del problema de la relación entre los pronombres y la categoría género. En lenguas como el español, en las que género de muchas palabras es (o parece ser) inmotivado, la forma de ciertos pronombres y elementos anafóricos responde al género del nombre (clasificación de elementos del lenguaje) mientras que otros pronombres oponen animado / inanimado respondiendo directamente a características del referente (clasificación nocional de entidades extralingüísticas):

$\begin{array}{cl}\text { (23)a. Vimos a Juan } & \rightarrow \text { Lo vimos // ¿A quién vimos? } \\ \text { b. Vimos a Maria } & \rightarrow \text { La vimos // ¿A quién vimos? } \\ \text { c. Vimos un muro } & \rightarrow \text { Lo vimos // ¿Qué vimos? } \\ \text { d. Vimos una pared } & \rightarrow \text { La vimos // ¿Qué vimos? }\end{array}$

En cambio, en los sistemas que contienen distinciones (¿de género?) sólo en los pronombres, éstas responden directamente a características del referente

(24) Inglés
a. We saw John $\rightarrow$ We saw him
b. We saw Mary $\rightarrow$ We saw her
c. We saw a wall $\rightarrow$ We saw it

Parece que en primer caso estamos ante una relación metalingüística (asimilable en todo lo esencial a la concordancia) entre la forma de un pronombre y las propiedades lingüísticas de un nombre que sirve de antecedente, mientras que en el segundo caso la variación de forma en el pronombre no se relaciona con 
propicdades lingüísticas del antecedente sino directamente con propiedades extralingüísticas del relerente. No hay por tanto concordancia y, en consecuencia, tampoco la catcgoría gramatical de género ${ }^{20}$.

De todo esto debemos concluir que es posible que los pronombres concuerden en género con su antecedente, y la prueba más clara la tenemos en los ejemplos del francés. Sin embargo, en sistemas pronominales con variación genérica detcminada semánticamente como el del inglés la clección de género remite a la designación y no a propiedades lingüísticas del nombre que sirve como antecedente. En consecuencia, no hay concordancia de género (y por tanto tampoco la calcgoría género). De todas formas, tanto en un caso como en otro, la variación genérica es importante en la identificación de cadenas referenciales en el discurso, pero por procedimicntos completamente diferentes: apuntando a características de referentes introducidos previamente o apuntando a las características de las palabras (los nombres) que hemos utilizado para referirnos a ellos.

\section{Conclusión}

Muchas lenguas utilizan un doble sistema de categorización relativo a las entidades designadas con el lenguaje: la categorización primaria por medio de los nombres y la cátegoriación secundaria realizada por los sistemas de clasificación nominal. La clasificación nominal se presenta en diferentes grados de gramaticalización. Un mayor grado de gramaticalización hace de la clasificación nominal un fenómeno cada vez más interno a la estructura linguística y, por tanto, repercute en una relación más fuerte con fenómenos estructurales como la concordancia y corrclativamente en la posibilidad de pérdida de motivación externa o scmántico-nocional, además de en el número total de clases reconocidas. Sin cmbargo, todo sistema de clasificación nominal conserva, al menos parcialmente, alguna base semántico-nocional. Los criterios de clasificación, fundados en cómo percibimos las entidades o en cómo interactuamos con ellas, dependen también de las lunciones de la clasificación nominal en la estructura y uso de las lenguas

La clasificación nominal proporciona un recurso lingüístico que puede ser útil cn la especilicación de referentes en el discurso o en su recuperación; pero eso no quicre decir que todo sistema pronominal recurra al señalamiento metalingüístico propio de la concordancia de género.

\footnotetext{
20) Martine (1956: 320) parece seguir este mismo razonaniento cuando dice que «el inglés, contrariamente a lo que pretenden ciertas gramáticas escolares no conoce el géncro gramatical, es decir, la servidumbre de empleo de ciertos temas de adjetivo o de pronombren. Como prueba contrasta el ingless ... the wath ... it... con cl francés la montre ... elle...
} 


\section{Referencias bibliográficas:}

Allan, Keith (1977): «Classifiers», Language 53/2, págs. 285-311.

Arias Barredo, Aníbal (1990): «Género gramatical y motivación semántica», ELUA 6, págs. 107-121.

Aronoff, Mark (1994): Morphology by itself. Stems and Inflectional Classes. Cambridge (Mass.), MIT Press.

Ashton, E.O. (1941): Swahili grammar. Londres, Longman.

Bybee, Joan (1993): «Mechanism of semantic change in grammaticization», conferencia leída en la III International Cognitive Linguistics Conference. Lovaina, julio 1993.

Carstairs-McCarthy, Andrew (1994): «Inflection classes, gender and the principle of contrast», Language 70/4, págs. 737-788.

Corbell, Greville (1989): «An approach to the description of gender systems», en D. Arnold et al. (eds.): Essays on Grammatical Theory and Universal Grammar. Oxlord, Clarendon Press, págs. 53-89.

Corbet, Greville (1991): Gender. Cambridge, Cambridge University Press

Cook, Dorothy M. \& L.L. Criswell: El idioma koreguaje (tucano occidental). Colombia: SIL 1993.

Craig, Colette (1992): «Classifiers in a functional perspective», en M. Fortescue, P. Harder y $\mathrm{L}$. Kristoffersen (eds.): Layered structure and reference in a functional perspective. Amsterdam, John Benjamins, págs. 277-301.

Craig, Colette (ed.)(1986): Noun Classes and Categorization. Amsterdam, John Benjamins

Crolt, William (1994): «Semantic universals in classifier systems», Word 45/2, págs. 147-171.

DeLancey, Scolt (1986): «Toward a history of Tai classifier systems», en C. Craig (cd.), págs. 437-452.

Denny, J. Peter (1976): «What are noun classifiers good for?», CLS 12, págs. 122132.

Denny, J. Peter y Chet A. Creider (1986): «The semantics of noun classes in Proto Bantu», en C. Craig (ed.), págs. 217-240.

Dixon, R.M.W. (1968): «Noun classes», Lingua 21, págs. 104-125. [Versión revisada en Dixon (1982), págs. 159-177.]

Dixon, R.M.W. (1982): Where have all the adjectives gone? and other essays in Semantics and Syntax. Berlin, Mouton.

Dixon, R.M.W. (1986): «Noun classes and noun classification in typological perspective», en C. Craig (ed.), págs. 105-112 
Downing, Pamela (1986): «The anaphoric use of classifiers in Japanese», en C. Craig (ed.), págs. 345-375.

Erbaugh, Mary S. (1986): «Taking stock: the development of Chinese noun classifiers historically and in young children», en C. Craig (ed.), págs. 399-436.

Greenberg, Joseph H. (1963): «Some universals of grammar with particular reference to the order of meaningful elements». En J. H. Greenberg (ed.) Universals of grammar. Cambridge (Mass.) y Londres, MIT Press, $1966^{2}$, págs. 73-113.

Grecnberg, Joseph H. (1978): «How does a language adquire gender markers?», en J.H. Grecnberg et al. (eds): Universals of human language. Vol. III: Word structure. Standford, Standford University Press, págs. 47-82.

Givón, Talmy (1984): Syntax. A Functional-Typological Introduction. Vol. I. Amsterdam, John Benjamins.

Hjelmslev, Louis (1938): «Essai d'une théorie des morphèmes» Actes du IVe Congrès International de Linguistes 1936. Copenhague, págs. 1140-151. [Versión española: «Ensayo de una teoría de los morfemas», en Ensayos Lingüísticos. Madrid, Gredos, 1972, págs. 200-217.]

Hjelmslev, Louis (1956): «Animé et inanimé, personnel et non-personnel», Travaux de l'lnstitut de linguistique (Paris) I, págs. 115-199. [Vcrsión española: «Animado c inanimado, personal y no personal», en Ensayos Lingiiísticos. Madrid, Gredos, 1972, págs. 278-329.]

Hopper, Paul J. (1986): «Some discourse functions of classifiers in Malay», en C. Craig (cd.), págs. 309-327.

Hopper, Paul J. y Elisabeth C. Traugott (1993): Grammaticalization. Cambridge, Cambridge Univ. Press.

Hundius, Harald y Ulrike Kölver (1983): «Syntax and semantics of numeral classifiers in Thai», Studies in Language 7/2, págs. 165-214.

Ibrahim, Muhammad Hasan (1973): Grammatical Gender. Its origin and development. The Hague, Mouton.

Johnsen, Lars (1992): «Number, gender and countability in Danish: An FG approach», en M. Fortescue, P. Harder y L. Kristoffersen (eds.): Layered structure and reference in a functional perspective. Amsterdam, John Benjamins, págs. 355-368.

Lchmann, Christian (1982): «Universal and typological aspects of agreement», en H. Seiler y F.J. Stachowiak (eds.): Apprehension. II: Die Techniken und ihr Zusammenhang in Einzelsprachen. Tubinga, Gunter Narr, págs. 201-267.

Lewis, G.L. (1967): Turkish Grammar. Oxford, Oxford University Press.

López García, Ángel (1989): Fundamentos de linguiística perceptiva. Madrid, Grodos 
Martinet, André (1956): «El género femenino en indoeuropeo: examen funcional del problema», Estudios de sintaxis funcional. Madrid, Gredos, 1978, págs. 317-331. [Versión original en francés en BSLP 52, 1956, págs. 83-95.]

Mel'c\&uk, Igor y Elie Bakiza (1987): «Les classes nominales en kirundi», Bulletin de la Société de Linguistique de Paris 82/1, págs. 283-341

Moreno Cabrera, Juan Carlos (1991): Curso universitario de lingiística general. I, Teoria de la gramática y sintaxis general. Madrid, Síntesis.

Renault, Richard (1987): «Genre grammatical et typologie linguistique», Bulletin de la Société de Linguistique de Paris 82/1, págs. 69-117.

Rosch, Eleanor (1978): «Principles of categorization», en E. Rosch y B.B. Lloyd (eds.): Cognition and Categorization. Hillsdale, Lawrence Erlbaum, págs. 2848 .

Seiler, Hansjakob (1986): Aprehension. Language, Object and Order. Part III: The Universal Dimension of Apprehension. Tubinga, Gunter Narr.

Wonder, John P. (1978): «Género natural, género gramatical», Español Actual 34, páss. 19-27.

Zubin, David y Klaus M. Köpcke (1986): «Gender and folk taxonomy: the indexical relation between grammatical and lexical organization», en C.G. Craig (cd.), págs. 139-180. 